\title{
Physical activity for coronary heart disease: cardioprotective mechanisms and effects on prognosis
}

\author{
Ruolo dell'attività fisica nella cardiopatia ischemica: \\ meccanismi cardioprotettivi ed effetti sulla prognosi
}

\author{
Domenico Scrutinio, Fabio Bellotto*, Rocco Lagioia, Andrea Passantino
}

\begin{abstract}
Physical activity for coronary heart disease: cardioprotective mechanisms and effects on prognosis. D. Scrutinio, F. Bellotto, R. Lagioia, A. Passantino.

A sedentary lifestyle is one of the five major risk factors for coronary heart disease (CHD) along with hypertension, abnormal values of blood lipids, smoking and obesity. After an acute myocardial infarction, risk factors continue to contribute synergically to the clinical progression and prognosis of CHD.

Regular physical exercise has been shown to improve exercise capacity and quality of life, to reduce symptoms and to decrease the risk of new coronary events in patients with CHD. Regular physical activity with its favourable ef-
\end{abstract}

\begin{abstract}
fects on coronary risk factors, endothelial dysfunction, inflammation, tendency to thrombosis, on autonomic tone and myocardial ischemia, may play a role in reducing the risk of new coronary events and death.

In view of the clinical benefits yielded and its well-documented cardioprotective mechanisms, regular physical activity should be regarded, by general practitioners and cardiologists, as a true and effective form of therapy for patients with CHD.
\end{abstract} nosis.

Keywords: physical activity, coronary heart disease, prog-

Monaldi Arch Chest Dis 2005; 64: 77-87.

IRCCS Fondazione "Salvatore Maugeri", Istituto di Cassano delle Murge (BA), Divisione di Cardiologia, * Dipartimento di Scienze Cardiologiche, Toraciche e Vascolari dell' Università di Padova, Italy

Corresponding author: Dr Domenico Scrutinio, IRCCS Fondazione Salvatore Maugeri, Istituto Scientifico di Cassano Murge, I-70020 Cassano Murge (Bari), Italy; E-mail address: dscrutinio@fsm.it

The World Health Organization has proposed three disability-death models according to the different patterns of disease evolution. ${ }^{1}$ In the first model, as in the case of polio, the onset of disease is associated with disability but not with a reduced life expectancy. In the other two models, the disease is associated with a premature death and either an intermittent (e.g. multiple sclerosis) or continuous disability (e.g. coronary artery disease). Actually, among middle-aged and elderly people, those with coronary heart disease (CHD) have substantially higher rates of disability that diminish their ability to perform everyday activities. ${ }^{2}$ As such, the goals of cardiac rehabilitation after myocardial infarction (MI) are to prevent disability resulting from CHD and to reduce the risk of subsequent coronary events and cardiac death through a program of prescribed exercise and interventions designed to modify coronary risk factors, including drug therapy and therapeutic lifestyle changes. ${ }^{3}$ In fact, several coronary risk factors such as physical inactivity, smoking, unhealthy diet, obesity, high plasma levels of triglycerides, low plasma levels of high-density lipoprotein and metabolic syndrome are strictly related to lifestyle. The recently published INTERHEART study has demonstrated that lack of exercise, smoking, abdominal obesity, and unhealthy diet account for a substantial proportion of the risk for an initial
MI worldwide, in both sexes of all ages, and in all regions; it is worth noting that the impact of physical inactivity on the risk of MI is comparable to that of diabetes. ${ }^{4}$ Consistently, in an Italian case-control study, the attributable risk for an initial MI for low occupational exercise at age 30 to 39 years was over $10 \% .5$ After an initial MI, risk factors continue to contribute synergically to clinical progression and prognosis of CHD. Appropriate use of cardioprotective drugs that have evidence-based efficacy for secondary prevention is also part of cardiac rehabilitation programs. ${ }^{6}$

Currently, changing lifestyle is an established therapeutic target in both primary and secondary prevention. The demonstration that interventions aimed at modifying lifestyle exert a protective effect against coronary events has recently been hailed as a milestone in the advance in knowledge of heart and vascular disorders over the last 25 years. ${ }^{7}$ The two seminal studies in this field are: the Stanford Three Community Study (1972) and the Martignacco Project Community Prevention Trial (1977); both showed a reduction in CHD risk owing to community-based interventions aimed at changing lifestylerelated risk factors such as physical inactivity, dietary habits and tobacco use. The importance of these studies is comparable, for example, to the studies demonstrating aspirin effective in preventing 
heart attacks and stroke (1970s), or Framingham's studies demonstrating the correlation between lipid profile and risk of death in young men (1987-1988), or the ISIS-2 trial (1988). ${ }^{7}$ It has been estimated that exercising, eating fruit and vegetables, and not smoking could lead to an approximately $80 \%$ lower relative risk of MI. ${ }^{7}$

A sedentary lifestyle is one of the five major risk factors for $\mathrm{CHD}$, along with hypertension, abnormal values of blood lipids, smoking and obesity. Estimates suggest that, worldwide, physical inactivity causes about $20 \%$ of cardiovascular disease and $22 \%$ of CHD.7 Currently, over $60 \%$ of the global population is not sufficiently active; this proportion is expected to increase in the future mainly as a result of growing urbanization. ${ }^{7}$ In Western Europe, an individual's energy expenditure per week in work, leisure and transport has been estimated to be less than 1.300 METs, indicating a low level of physical activity. ${ }^{7}$ Moreover, the time spent seated each week, another index of inactivity, is as high as 30 to 42 hours. In Italy, $34 \%$ of men and $46 \%$ of women are physically inactive (Progetto Cuore, 2004. www.iss.it).

Exercise is linked to longevity independently of genetic factors. Even at an older age, physical activity can significantly reduce the risk of coronary events, diabetes, hypertension and obesity, and improve lipid profile. ${ }^{7}$ It has been calculated that, despite documented evidence of the benefit of physical activity in preventing cardiovascular diseases, more than a quarter of a million individuals in the United States die each year due to a lack of regular exercise. ${ }^{7}$ The risk of CHD can be reduced by approximately $30 \%$ if one does more than 150 minutes of moderate physical activity or 60 minutes of vigorous physical activity weekly. ${ }^{7}$

\section{Physical inactivity as a therapeutic target in secondary prevention}

The role of regular physical exercise in preventing cardiovascular diseases in the population has recently been emphasized in a publication endorsed by the World Health Assembly (WHO Global Strategy on Diet, Physical Activity and Health, 2004). The beneficial effects of exercising in the setting of secondary prevention have been stressed in the European Guidelines on Cardio-Vascular Disease Prevention ${ }^{8}$ and by the American College of Cardiology/American Heart Association Guidelines for the Management of Patients with ST-Elevation Myocardial Infarction. ${ }^{9}$ On the basis of the assessment of risk, ideally with an exercise test to guide the prescription, all patients recovering from ST-elevation MI should be encouraged to exercise a minimum of 30 minutes at least 3 or 4 times a week (preferably daily), and should furthermore increase their daily activities. ${ }^{9}$ Instructions on daily exercise, on the strenuous activities permitted and those that ought to be avoided should be provided. These recommendations are primarily based on the results of cohort longitudinal studies that have provided overwhelming evidence of the close association between regular physical activity and the reduced risk of new coronary events. The very large overall sample size (several hundred thousand subjects and patients) and the consistency of results in the various studies provide persuasive evidence of causality. Unfortunately, despite significant evidence of benefit and despite guidelines recommendations, nearly $80 \%$ of patients remain physically inactive after MI. Moreover, only a minority of patients receive information on lifestyle. In this article, we will review the results of the studies investigating the effects of physical activity on prognosis of patients with CHD and the possible cardioprotective mechanisms.

\section{Effects on prognosis}

It is a well-known fact that regular physical exercise increases exercise capacity, improves quality of life and reduces symptoms in CHD patients. Moderate-to-high intensity aerobic conditioning (at 65 to $85 \%$ of the maximal measured heart rate) can increase maximal exercise tolerance by 30 to $50 \%$ and peak exercise oxygen consumption by 5 to $20 \% .3,10$ The ability to perform daily activities is also significantly improved. ${ }^{11}$ This also applies to patients with heart failure in whom aerobic conditioning results in improvements in neurohumoral and skeletal muscle abnormalities, exercise capacity, and quality of life. 12

The relation between exercise and risk of coronary events has been addressed by numerous epidemiologic and secondary prevention studies. All studies have consistently demonstrated a close relation between regular physical activity and reduced risk of mortality and morbidity from CHD. These findings, along with studies demonstrating biologically plausible cardioprotective mechanisms, provide strong evidence that regular physical activity, of at least moderate intensity, reduces the risk of CHD. 6 The greatest gains, in terms of survival, are achieved when an individual moves away from a sedentary lifestyle and becomes moderately active; less is gained when an individual goes from moderately active to very active. 13,14

Epidemiologic studies. In the Health Professionals' Follow-up Study, 15 involving 44,452 US men, the association between amount, intensity and type of physical exercise in relation to risk of CHD was assessed. A significant inverse dose-response relationship between total physical activity and risk of CHD was demonstrated. Moreover, average exercise intensity was associated with a reduced risk independent of the MET-hours spent on physical activity. A novel finding to emerge from this study was the significant reduction in CHD risk from resistive-type activities (i.e. weight training and use of strength machines). The authors concluded that increasing the total volume of activity, increasing the intensity of aerobic exercise from low to moderate (4 to 6 METs) and from moderate to high, and adding weight training to the exercise program are the most effective strategies to reduce the risk of CHD in men. In a prospective study of 73,743 postmenopausal women ranging from 50 to 79 years of age, a strong, graded, inverse association between increasing physical-activity score and risk of both coronary events and total cardiovascular events was demonstrated. 16 Both walking and vigorous exercise were associated with a substantial reduction in the incidence of cardiovas- 
cular events irrespective of race, age, and body mass index. Conversely, prolonged sitting determined increased cardiovascular risk.

Current recommendations prescribe at least moderate-intensity physical activity, requiring $\geq 3$ METs for $\geq 30$ minutes almost daily, generating $\approx 1000 \mathrm{Kcal} / \mathrm{wk}$. Since defining intensity using an absolute scale in METs may be limited as it neglects variations in physical fitness, Lee et al. examined the association between relative intensity of physical activity and the risk of developing CHD in 7,337 men using the Borg Scale as a measure of subjective rating of exertion. 17 A significant inverse correlation between a "somewhat strong" intensity of physical activity (4/10 at the Borg Scale) and the risk of CHD was demonstrated. Finally, the association between leisure-time physical activity and lower allcause mortality 18 and lower morbidity and mortality rates from $\mathrm{CHD}^{19}$ in middle-aged and older men was also demonstrated. In older men, even light activities were shown to be beneficial. 19,20

Secondary prevention studies. A recently published meta-analysis involving 8,940 patients with CHD provides data on the effects of exercise-based rehabilitation on all-cause and cardiac mortality. 21 Of the subjects, $67 \%$ were post-myocardial infarction patients. Compared with usual care, cardiac rehabilitation was associated with a statistically significant reduction in all-cause mortality $(-20 \%)$ and cardiac mortality (-26\%). A statistically insignificant $21 \%$ reduction in risk for nonfatal MI was also observed. The effect on mortality was independent of CHD diagnosis, dose of exercise intervention, duration of follow-up and trial publication date. Data from a limited number of studies included in this meta-analysis also showed favourable changes in total cholesterol, triglyceride levels, systolic blood pressure and lower rates of self-reported smoking in patients who received exercise therapy. Witt and coworkers examined the association of participation in cardiac rehabilitation after MI with survival in a community-based cohort of 1,821 patients with incident MI.22 Approximately half of the patients participated in a cardiac rehabilitation program that included supervised exercise, counseling and education sessions. Because of marked differences between participants and non-participants, propensity scores were used to adjust for the characteristics associated with participation in cardiac rehabilitation. A logistic regression model was fitted so as to predict whether a person would participate in cardiac rehabilitation as a function of 16 variables including demographic and clinical characteristics, use of reperfusion therapy, if a cardiologist was the primary attending physician and measurement of the ejection fraction within 30 days after MI. Participants had a marked survival advantage over non-participants with a three-year survival of $95 \%$ compared with $64 \%$ for the non-participants. After adjustment for the propensity score, a $56 \%$ improvement in survival and a 28\% reduction in the risk of recurrent MI was still appreciable. In a study involving $772 \mathrm{men}$ with established CHD, Wannamethee and co-workers investigated the relations between physical activity, types of physical activity, changes in physical activity and all cause-mortality. ${ }^{23}$ Light to moderate levels of physical activity were associated with a significant reduction in risk of all-cause mortality and cardiovascular mortality, irrespective of age $<$ or $\geq 65$ years. Benefits were attained even through light forms of physical activity such as walking $>40$ $\mathrm{min} / \mathrm{d}$, gardening and recreational activity. In a posthoc analysis of the Cholesterol And Recurrent Events trial, regular moderate exercise (e.g. brisk walking, riding a stationary bicycle, moderate gardening or housework three or more times per week at baseline) was independently associated with a $30 \%$ lower risk of heart failure requiring hospitalization and of the combined end-point of heart failure and death, even after adjusting for age and comorbid illnesses. ${ }^{24}$ The clinical effects of exercise training after percutaneous coronary angioplasty were investigated by Belardinelli and co-workers. 25 Maximal exercise capacity, as assessed by peak exercise oxygen consumption determination, and quality of life were significantly improved. In the exercise-training patient group, a statistically and clinically significant reduction in risk of cardiac events and hospital readmissions of around $60 \%$ was observed during a 33-month follow-up. In a provocative study, Hambrecht and co-workers randomized 101 patients with stable coronary artery disease to percutaneous coronary angioplasty with stenting or a 12-month program of daily 20 minute sessions on a bicycle ergometer at $70 \%$ of the symptom-limited maximal heart rate. ${ }^{26}$ Aerobic conditioning resulted in greater event-free survival (88 vs. $70 \%$ ) and exercise capacity at lower costs, notably owing to reduced hospitalizations and repeat revascularizations. According to the authors, these data document that exercise training as a lifestyle intervention can be an alternative approach to an interventional strategy in selected, highly motivated patients with stable CHD. Obviously, for most patients with stable CHD, percutaneous coronary angioplasty remains the therapy of choice but, without an aggressive lifestyle intervention that includes daily physical exercise, it should be viewed as a suboptimal therapeutic strategy. 25,26 Finally, Giannuzzi et al demonstrated that patients with left ventricular dysfunction after MI may benefit from physical training without any additional clinical deterioration or other adverse events. ${ }^{27}$ More importantly, their data indicate that, for post-infarction patients with poor left ventricular function, long-term exercise training may attenuate the unfavourable remodelling process and even improve over time both the regional and global left ventricular function.

\section{Cardioprotective mechanisms}

The mechanism of the cardioprotective effects of regular physical activity in patients with CHD is multifactorial. The favourable results of physical activity on coronary risk factors, endothelial dysfunction, inflammation, tendency to thrombosis, autonomic tone and myocardial ischemia may all play a part in reducing the risk of death and new coronary events. ${ }^{2}$ Currently, some of these effects, such as those on endothelial dysfunction and inflammation, are being actively investigated. 


\section{- Coronary risk factors}

Luigi Cavalli-Sforza recently opened a seminar on human genetics in New York with the following sentence: "To understand the present, you have to understand history and to understand biology you have to understand evolution because evolution is the history of biology". In the process of evolution, all living creatures including humans have constantly and progressively adapted their physical and intellectual capacity to the particular conditions of a specific area and period. But the development of mankind has an exclusive peculiarity: its evolutive process has been expanded and enriched by rational behaviour, a unique intellective development of the human brain. Yet, man's intellect has not always worked towards improving his health: in fact, urbanization has made people less physically active. This factor, in tandem with an unhealthy diet, has brought about a secondary effect: the occurrence of a constellation of metabolic abnormalities that include glucose intolerance (type 2 diabetes, impaired glucose tolerance, or impaired fasting glycaemia), insulin resistance, central obesity, dyslipidaemia, and hypertension. All these well-documented conditions have proven to be risk factors for cardiovascular disease and can serve to explain the overwhelming increase of atherosclerotic diseases in the civilized world. The hypothesis that physical activity alone can reduce or even eliminate the impact of each single risk factor will be the focus of the next section.

Metabolic syndrome. In a recent review on the metabolic syndrome, 28 an extremely frequent condition that embraces all the above-mentioned disorders, physical inactivity appears to be the most important etiologic factor, whereas regular and sustained physical exercise appears to be the most promising, and sometimes even the most adequate, therapeutic intervention. ${ }^{29}$ Changing lifestyle is fundamental in treating metabolic syndrome. The newly modified lifestyle routine must include a nutrition plan and exercise therapy in order to achieve a net negative energy balance, with the ultimate goal of decreasing both body weight and insulin resistance. ${ }^{30}$ Prevention should start as early as in childhood and should include: a healthy diet, adequate daily physical activity, annual measurement of weight and height, and regularly monitored blood pressure, although most patients will require lifelong lifestyle modifications and, sometimes, additional pharmacologic therapy. ${ }^{31}$ Admittedly, in a well-regulated lifestyle, exercise has proven to lead to notable benefits both in primary and secondary prevention, 32,33 but the possible advantages of physical activity per se in the control of each single risk factor, independently of the net clinical advantage in terms of mortality reduction or event free survival, have attracted less attention.

Diabetes. Increased physical activity is not only associated with a lower risk of cardiovascular disease in the general population but it also benefits those with type 2 diabetes. In the case of diabetic women, for instance, greater physical activity, that includes regular walks, has led to a substantially lower risk of cardiovascular events. ${ }^{34,35}$ Moreover, epidemiological studies have demonstrated that physical activity done at different grades of intensity, ranging from vigorous to leisure-time, can protect against the development of type 2 diabetes. $36-39$ Both the Finnish Diabetes Prevention Study 40 and the United States Diabetes Prevention Program 41 have proven that good nutrition flanked by exercise plays a significant role in reducing the progression from impaired glucose tolerance to type 2 diabetes. A recent randomized, controlled trial on the role of leisure-time physical activity in preventing type 2 diabetes was conducted on 487 men and women with impaired glucose tolerance. 42 During the 4.1year follow-up, 107 new cases of diabetes arose; post hoc analyses showed that those who increased from moderate to vigorous physical activity were less likely, by $63-65 \%$, to develop diabetes compared to controls. Hu et al. prospectively evaluated 3,708 Finnish patients with type 2 diabetes. Physical activity, smoking, blood pressure, height, weight and serum cholesterol were determined at baseline and cox proportional hazard models were used to estimate single and joint effects of physical activity and other cardiovascular risk factors affecting mortality. ${ }^{43}$ In the 18.7 mean years of follow-up, 906 cardiovascular related deaths were recorded. Moderate to intensive physical activity was associated with a reduced total and cardiovascular mortality. The beneficial effects of physical activity were associated with a lower risk among diabetic patients, regardless of the levels of BMI, blood pressure, total cholesterol, and smoking. Notably, aerobic leisuretime physical activity has been shown to improve not only health but has also turned out to be financially advantageous, supporting the need for - and convenience of - instituting physical activity programs as an essential part of therapy for type 2 diabetes. 44

Hypertension. Hypertension and physical inactivity are extremely common and very often associated conditions. The prevention of hypertension should include lifestyle modifications in which exercise is the hub of the control and even the treatment. The proposed mechanism in lowering blood pressure through exercise includes the adaptation of both vascular and neurohumoral systems that leads to a decrease of total peripheral resistance and catecholamines. 45 Studies have documented the way endurance exercise training significantly reduces blood pressure, even though - similarly to other risk factors individual responses are highly variable. $46-48$ In fact, an analysis of the positive effects that training alone has on blood pressure is very difficult as exercise implies many other different effects like lipid and glucose control and weight reduction. Emerging data suggest that there is a genetic link between the intensity of blood pressure reduction and acute and chronic exercise: the variability of results in individuals was investigated by Hamberg and coll. 49 Apparently, ACE, apoE, and LPL genotypes can identify hypertensive individuals whose blood pressure and cardiovascular risk can be significantly reduced with training. Such genotype-dependent responses can help to better understand the mechanisms by which endurance exercise can improve blood pressure and plasma lipid levels, especially in genetically 
favoured individuals. Even if pharmacological therapy is frequently indicated for physically active people to lower blood pressure at rest and during exertion and to decrease peripheral resistances, endurance exercise, supplemented by resistance exercise, remains a cornerstone therapy for primary prevention, control and treatment of hypertension. 45

Obesity. Excess body fat and sedentariness are independent and almost always linked risk factors for cardiovascular disease. The relation between obesity - particularly when visceral - and cardiac mortality is complex but, apparently, physical activity has an independent effect on cardiovascular risk; obesity, on the other hand, increases the risk partially through modification of other factors. 50 In fact, BMI, percent body fat and waist circumference are strictly associated with other metabolic factors and they are strong predictors of cardiovascular risk even after correction for aerobic fitness and age. ${ }^{51} \mathrm{It}$ has been postulated, but with inconsistent evidence, that physical activity can eliminate the effect of obesity. In overweight people, increasing general physical activity has been hypothesized to play a major role in weight loss, but only minor losses in body mass have been reported with exercise in the absence of dieting. 52 It is also important to note that the combination of diet and exercise provides greater losses in body mass compared with either diet or exercise alone. 53 To examine whether higher levels of physical activity can attenuate the association between body mass index and mortality, the Nurses' Health Study was extended to a 24 year follow-up and a J-shaped relationship between BMI and overall mortality was observed in age-adjusted analyses. ${ }^{54}$ However, after restricting the analysis to non-smokers, a direct monotonic relationship was found, with the lowest mortality among women with a BMI of less than 23.55 Both BMI and level of physical activity were important and independent predictors of mortality in this study: even a moderate weight gain during adulthood was, in fact, associated with an increase in mortality, independently of the physical activity level. In accordance with Wilmore, we can conclude that, to fight obesity, greater focus must be placed on prevention in childhood and adolescence, but even obese adults who increase their level of exercise will have an improved life expectancy with lower future risks of weight gain that translate into a healthier condition. 56

Dyslipidaemia. As recently reaffirmed by the Adult Treatment Panel III (ATP III) of the National Education Program on cholesterol management, therapeutic lifestyle changes remain an essential modality for lipidaemic control, particularly for those who have lifestyle-related risk factors like obesity, elevated triglycerides, low HDL-C or metabolic syndrome. 57 Walking is a "light" form of physical activity that results particularly appropriate for the vast majority of people; it is clearly a low-cost, low-risk intervention but its usefulness, alone, in reducing lipoproteins is not well established: a metaanalysis dealing specifically with the effects walking has on lipids and lipoproteins in adults produced interesting data. ${ }^{58}$ Fifteen randomized controlled trials were included and evaluated with a total of 1,176 subjects (692 walkers and 484 controls); the vast majority of these studies followed the guidelines of walking briskly for 30 minutes or more at least 5 days a week. While all changes in lipids and lipoproteins suggest a benefit, a statistically significant decrease was limited to LDL-C (by 5\%) and TC/HDL-C (by 6\%), independently of changes in body composition; moreover, the greater reduction was associated with increasing age as well as increases in $\mathrm{VO}_{2 \max }$ with the better results in postmenopausal women. Any exercise that is more vigorous than walking appears advantageous in reducing lipid levels: the effect of different intensity of exercise on a variety of lipid and lipoprotein variables was analysed in a prospective randomized trial.59 The higher-amount of intensity exercise compared with the lower had the most positive effect and it always resulted superior to the control group. It is worth noting that a high amount of high-intensity exercise significantly reduced small LDL-C and increased the average size of LDL particles, showing a clearly beneficial effect on the HDL-C in the same group, whereas the lower amount of exercise had non-significant effects on these variables. Moreover, in the high-amount high-intensity, low-amount high-intensity, and low-amount moderate-intensity groups, a marked improvement in triglyceride and VLDL concentrations was noted.

To sum up it can be claimed that moderate, regular physical activity not only positively affects mortality and morbidity but also reduces and even eliminates the relevance of the most important risk factors for cardiovascular disease such as diabetes, hypertension, obesity and dyslipidemia.

\section{- Endothelial dysfunction}

As a result of the chronic inflammatory process initiated by coronary risk factors, the endothelium may adopt a phenotype that facilitates vasoconstriction, thrombosis, inflammation and proliferation in the arterial wall thus contributing to the development, progression and clinical manifestations of atherosclerosis. ${ }^{60,61}$ The central way endothelial dysfunction contributes to atherosclerosis/atherothrombosis is a decreased bioactivity of nitric oxide (NO). In the endothelium, NO is synthesized from L-arginine under the influence of endothelial nitric oxide synthase (eNOS). Decreased levels of NO promote vasoconstriction, leucocyte adherence, platelet activation, mitogenesis, oxidation, thrombosis, impaired coagulation, and vascular inflammation. ${ }^{61} \mathrm{In}$ addition, decreased NO production may contribute to plaque destabilization, facilitating rupture and inciting acute coronary syndromes. ${ }^{62}$ Moreover, NO has been suggested to be a key modulator of angiogenesis, 63 an important compensatory mechanism to long-standing ischemia, and to inhibit endothelial cell apoptosis. 64 Thus, in states of diminished NO bioactivity, apoptosis may be facilitated and angiogenesis inhibited.

The causality between endothelial dysfunction and clinical manifestations of atherosclerosis has been suggested by some retrospective and prospective outcome studies involving patients with CHD or hypertension referred for cardiac catheterization or 
undergoing vascular surgery. 61 These studies, that used the endothelium-dependent flow-mediated vasodilatory capacity measured at the level of either coronary or brachial vascular bed as a marker of endothelial function, consistently support the notion that severity of endothelial dysfunction is related to cardiovascular risk. $61,65,66$ The mechanisms by which endothelial dysfunction may contribute to specific cardiovascular events have recently been reviewed by Lerman and Zeiher.66

In the prospective study of Heitzer and co-workers, conducted on 281 patients with stable coronary artery disease, the forearm blood flow vasomotor response to acetylcholine independently predicted the combined end-point of cardiovascular death, MI, stroke, coronary revascularization procedures and peripheral bypass. ${ }^{67}$ Lately, it has also been shown that systemic endothelium-dependent vasoreactivity, measured within 5 days of the index event, predicts recurrence of instability and cardiovascular event rates in patients with acute coronary syndromes. 68 Reduced vasodilator response to acetylcholine and troponin $\mathrm{T}$ were the only significant independent predictors of poor outcome, even after adjustment for traditional risk factors, concurrent medication, invasive treatment strategy, and C-reactive protein levels. Importantly, the recovery of systemic endothelial function within 8 weeks after the index measurement was associated with event-free survival. Furthermore, among the traditional cerebrovascular disease-related risk factors, the presence of coronary endothelial dysfunction resulted to be the single strongest independent predictor of cerebrovascular events in 503 patients without obstructive coronary artery disease. ${ }^{69}$ Finally, systemic endothelial dysfunction was independently and closely associated with late in-stent coronary restenosis in 141 consecutive patients who had elective and successful percutaneous coronary intervention. ${ }^{70}$

The results of such studies provide compelling scientific evidence that the presence of endothelial dysfunction relates to the risk of cardiovascular events 66 and lead to the hypothesis that reversing endothelial dysfunction reduces the risk. Although this hypothesis has not been tested directly in prospective randomized clinical trials, there are several interventions that can improve endothelial function and reduce cardiovascular risk. 61 Exercise training is one such modality. It has been demonstrated effective in improving endothelial function in healthy sedentary subjects and in hypertensive, heart failure and CHD patients. ${ }^{61}$ The seminal study by Hambrecht and co-workers demonstrated that exercise training improves endothelium-dependent vasodilation, both in epicardial coronary vessels and resistance vessels, and coronary blood-flow reserve in patients with CHD. 71 This beneficial effect can be produced by moderate aerobic training but rapidly disappears with detraining. 72

The mechanisms responsible for the exercise-induced improvement in endothelial function have been elucidated. Experimental studies suggested shear stress-induced changes in eNOS expression/ activity to be possible mechanisms that lead to improved endothelial function. ${ }^{74}$ These mechanisms have been confirmed in humans. Hambrecht and co- workers demonstrated that, in trained CHD patients, exercise-induced improvement in endothelium-dependent vasodilatory capacity is accompanied by a 2-fold increase in eNOS mRNA expression. 75 Nitric oxide production depends also on eNOS phosphorylation. Among the five potential eNOS phosphorylation sites, the Ser1177 residue seems to function as a sensor of shear stress. The authors found a 4-fold higher Ser1177-eNOS phosphorylation level in CHD patients after 4 weeks of exercise training compared with sedentary controls. 75 The proportion of eNOS phosphorylated on Ser1177 was closely correlated with the exercise training-induced improvement of endothelial function. As far as the intracellular signalling pathway of increased eNOS phosphorylation is concerned, a close correlation between activated Akt, a protein kinase which phosphorylates eNOS at Ser1177 in endothelial cells subjected to fluid shear stress, and eNOS phosphorylation was demonstrated. ${ }^{75}$ Improvement in endothelial dysfunction may also result from a decreased breakdown of NO by reactive oxygen species (ROS). In CHD patients, the Ang II-NAD(P)H oxydase system, whose expression and activity are regulated by the dominant influence of the Ang II type 1 receptor $\left(\mathrm{AT}_{1}-\mathrm{R}\right)$ expression on cell surface, 76 is a major source of ROS generation. 77 Hambrecht's research group has recently demonstrated that, in patients with symptomatic stable CHD, exercise training decreases ROS generation via the downregulation of $\mathrm{AT}_{1}-\mathrm{R}$ and subsequently reduced expression and activity of $\mathrm{NAD}(\mathrm{P}) \mathrm{H}$ oxydase. 78 A negative linear correlation between endothelial function and $\mathrm{NAD}(\mathrm{P}) \mathrm{H}$ activity or ROS production was documented thus supporting the concept that exercise training-induced downregulation of ROS production leads to improved endothelial function. In short, in CHD patients, exercise training improves endothelial dysfunction through both an increase in shear stress-induced eNOS expression and phosphorylation and a decrease in premature breakdown of NO by ROS resulting in enhanced $\mathrm{NO}$ availability.

A novel aspect of the relation between exercise training and the endothelium regards the effects of exercise training on bone marrow-derived endothelial progenitor cells (EPCs). Vascular function may be significantly modulated by EPCs. These circulating cells have experimentally been shown to promote endogenous vascular repair, to improve endothelial function and to increase ventricular function after MI.79 Moreover, EPCs have also been implicated in the enhancement of regional perfusion in the ischemic myocardium; EPCs which "home" into ischemic regions may form entirely new vessels by cell division and differentiation into endothelial cells, thus promoting vasculogenesis. 80 Atherosclerotic risk factors reduce the EPC number and function. 79 Moreover, reduced levels of circulating EPCs have recently been shown to independently predict cardiovascular events in patients with stable or unstable CHD. ${ }^{81}$ After adjustment for disease activity and overall risk factor load for CHD, low number of EPCs was associated with a significantly, $\approx 4$-fold increased risk of suffering a cardiovascular event during follow-up. According to the authors, these findings support an important role for 
endogenous vascular repair to modulate the clinical course of CHD.

Exercise training may increase the number of circulating EPCs, via a partially NO-dependent antiapoptotic effect.82 After a 4-week exercise bicycle ergometer training program, moderate muscle strength training and walking, a statistically significant $78 \%$ increase in EPCs number in the peripheral blood was observed. 82 As endogenous stimuli such as tissue ischemia have been demonstrated to promote the mobilization of EPCs, Sandri and coworkers investigated whether brief episodes of ischemia are required during exercise training to increase the EPCs count or promote homing of EPCs into vascular network. 83 The authors concluded that, though symptomatic tissue ischemia seems to be a prerequisite for EPCs mobilization, subischemic ergometer training in patients with CHD may lead to an improved EPCs integration into the endothelial network, believed to be important for endogenous vascular repair and during growth of new blood-vessels. ${ }^{79,80}$

\section{- Inflammation}

Inflammation plays a fundamental role in mediating initiation, progression and thrombotic complications of atherosclerosis 84 Among the various markers of inflammation, C-reactive protein (CRP) has been the most extensively studied. C-reactive protein has not only been recognized as a marker of atherosclerosis/coronary events but is also a mediator of the disease because it contributes to the pathogenesis of lesion formation, plaque rupture and coronary thrombosis by interacting with and altering the endothelial cell phenotype. ${ }^{85}$ High-sensitivity (Hs) CRP has been found to be a strong predictor of short- and long-term occurrence of atherothrombotic events in acute coronary syndromes, including ST-elevation MI, and in stable CHD. 86 Also relevant to the cardiac rehabilitation setting is the observation of a higher recurrence rate among patients with elevated levels of Hs-CRP before percutaneous coronary angioplasty or coronary artery bypass surgery. 86,87 In a recently published study, Hs-CRP has also been found to be associated with rapid angiographic coronary artery disease progression in patients with chronic stable angina. $88 \mathrm{Hs}-\mathrm{CRP}$, matrix-metalloproteinase-9, and soluble intercellular adhesion molecule 1 remained independently associated with rapid coronary artery disease progression even when the presence of complex lesions was introduced into the multivariate model. This finding suggests a significant role of inflammatory mechanisms in atheromatous plaque vulnerability and rapid coronary artery disease progression. Physical activity, weight loss and statins are shown to reduce serum levels of CRP. 85

The effects of physical activity on serum CRP and inflammatory markers in healthy subjects and the mechanisms underlying its anti-inflammatory effect have recently been reviewed by Kasapis and Thompson. 89 Cross-sectional comparisons and longitudinal exercise training studies demonstrate an inverse relationship between regular physical activity and the serum concentration of inflammatory markers, even after adjustment for confounders. 89 Milani and co-workers investigated the effects of a cardiac rehabilitation and exercise training program on Hs-CRP levels in 235 patients with CHD (72\% coronary angioplasty, $19 \%$ bypass surgery, and $28 \%$ MI). ${ }^{90}$ After three months, median levels of Hs-CRP decreased significantly from 3.4 to $2.0 \mathrm{mg} / \mathrm{dl}$ in the rehabilitation group but not in the control population. Even the rate of patients with elevated Hs-CRP $(\geq 3 \mathrm{mg} / \mathrm{l})$ decreased significantly in the rehabilitation group from $54 \%$ to $38 \%$ but remained unchanged in the control group. Similar significant reductions in Hs-CRP occurred in the rehabilitation patients regardless of statin therapy or weight loss, suggesting that the effects of exercise training are incremental over those therapies proven to be effective in reducing Hs-CRP. In a randomized trial of 39 patients with symptomatic peripheral arterial disease, both CRP and serum amyloid-A levels were significantly reduced after 3 and 6 months of supervised exercise compared to controls. 91 This anti-inflammatory response may contribute to the beneficial effects of regular physical activity.

The way exercise training reduces inflammation and suppresses CRP levels is not well defined since aerobic conditioning is also related to some confounders that are independently associated with CRP levels. ${ }^{89}$ Regular physical activity is thought to reduce CRP levels by multiple direct and indirect mechanisms including a decrease in cytokine production by adipose tissue, skeletal muscles, endothelial and blood mononuclear cells, improved endothelial function and insulin sensitivity, and a possible antioxidant effect. 89 Moreover, the expression of adiponectin (an adipocyte-specific secretory protein exerting cardioprotective effects through its anti-inflammatory properties) from visceral adipose tissue is impaired in obesity. ${ }^{92}$ Weight reduction, promoted by regular physical activity, might indirectly contribute to improve adiponectin secretion.

\section{- Haemostatic effects}

The effects of regular physical activity on thrombogenic factors were investigated in several intervention studies, prospective randomized trials and large epidemiologic studies. ${ }^{93,94}$ Some of these studies, however, have provided variable or fragmentary results due to the lack of a comprehensive evaluation of both haemostatic and fibrinolyitc variables, the populations studied, variations in exercise training protocol and the lack of standardization in the various analytical methods applied, particularly in the assessment of platelet function. 94

Notwithstanding, there is persuasive and consistent evidence that regular physical activity enhances fibrinolytic capacity, as indicated by lower PAI-1 and higher tPA activity levels, regardless of age or exercise intensity. 93 More recently, Smith and co-workers for the first time have shown that a moderate, homebased aerobic exercise training program can not only prevent but fully restore the age-associated loss in the capacity of the vascular endothelium rapidly and locally to release t-PA in previously sedentary individuals, a critical aspect for effective endogenous fibrinolysis. ${ }^{95}$ Importantly, the decline in the capaci- 
ty of the endothelium to release tPA did not appear to be a consequence of primary ageing, but rather a byproduct of a sedentary lifestyle. This study provides important additional information on the effects of aerobic conditioning on endothelial function.

Although an inverse relationship between regular physical activity and plasma fibrinogen levels was shown in some studies, 94 the results related to the changes in fibrinogen level in response to regular physical activity are not uniform. In addition, the reported effects of physical exercise on platelet aggregation and activation are rather variable most likely due to the considerable methodologic difficulties in measuring platelet reactivity. 93

The divergent effects of short-term versus regular exercise on thrombotic risk also need consideration: while both regular physical activity and shortterm strenuous exertion induce a transient enhancement of fibrinolytic capacity, blood coagulation is persistently activated by short-term strenuous exertion but unaffected or possibly reduced by regular physical activity. ${ }^{93}$ In addition, short-term strenuous exertion induces in vivo a transient increase in agonist-induced platelet aggregation and secretory activity in sedentary subjects; 93,94 this pro-thrombotic response to heavy exertion is prevented by longterm physical exercise but not by aspirin. ${ }^{93}$ Thus, unaccustomed strenuous exertion can potentially lead to acute coronary thrombosis in sedentary subjects with pre-existing coronary artery disease whose fibrinolytic capacity may be impaired due to endothelial dysfunction. 93,94

\section{- Autonomic function}

Alterations in cardiac autonomic balance after an acute myocardial infarction (i.e. enhanced sympathetic and reduced parasympathetic activities) are associated with an increased risk of sudden cardiac death. Clinical studies have demonstrated that postmyocardial infarction patients with either a reduced heart rate variability or a depressed baroreflex sensitivity after MI have a substantially higher risk of cardiac death. 96,97 The patients with the greatest alterations in the markers of sympathovagal imbalance also have the greatest risk of a sudden death. Exercise training is a well-established method capable of eliciting changes in cardiac autonomic balance after MI, potentially resulting in a decreased susceptibility for the development of malignant ventricular arrhythmias and sudden death.98 A hospitalbased exercise training program consisting of 2 daily 30-minute sessions of stationary cycling 6 times a week for 2 weeks significantly increased baroreflex sensitivity and heart rate variability in 97 male CHD patients, with or without MI, who were recruited after myocardial revascularization surgery. 99 Malfatto and co-workers also proved that the association of exercise training and $\beta$-blockers induces a more favourable sympathovagal balance that persists in the long-term. 100 Minimal additional benefits on cardiac autonomic function from a hospital-based formal exercise training program were instead observed by Leith and co-workers in patients with an uncomplicated MI as compared to controls, even though significant changes in baroreflex sensitivity and heart rate variability actually occurred in the formal exercise training group. ${ }^{101}$ It should however be noted that the control patients were not inactive but were advised to follow a home walking program that, in motivated patients, can produce a significant training effect. Thus, the lack of a true sedentary control group may have flawed the differences between groups.

Although a direct causal link between exerciseinduced alterations in the cardiac autonomic regulation and a reduced sudden death rate has yet to be established, ${ }^{99}$ there is scientific evidence that aerobic conditioning can achieve a positive impact on the markers of cardiac autonomic balance related to a higher risk of sudden cardiac death after MI and hence may potentially reduce the risk of this catastrophic event.

\section{- Myocardial ischemia}

It is a well-known fact that aerobic conditioning also has antiischemic effects. Exercise training can reduce the ischemic response at a given submaximal work rate by decreasing rate-pressure product and myocardial oxygen demands during exertion.6,102 Patients with CHD may thus attain a higher level of physical work before reaching the level of myocardial oxygen demand resulting in ischemia. Some studies have also reported an exercise training-induced decrease in the ischemic response at a given rate-pressure product, suggesting an improved myocardial oxygen supply. 6 Possible explanations of this finding are: improved endothelium-dependent coronary vasodilation leading to enhanced myocardial perfusion, 75 increase in coronary artery collateral, 103 and improved coronary artery compliance or elasticity. ${ }^{6}$

\section{Conclusions}

In conclusion, there is strong evidence that both healthy sedentary subjects and patients with stable coronary heart disease can benefit from regular physical activity since it reduces the risk of a first or recurrent cardiovascular event. Physical activity yields beneficial effects by positively interferring with nearly all the mechanisms mediating the progression and clinical manifestations of atherosclerosis. In view of its unquestionable clinical benefits and biologically plausible well-documented cardioprotective mechanisms, regular physical activity should be considered by general practitioners and cardiologists as a true and effective form of therapy for patients with coronary heart disease.

\section{Riassunto}

Uno stile di vita sedentario è uno dei cinque maggiori fattori di rischio per cardiopatia coronarica, insieme con l'ipertensione, la dislipidemia, il fumo e l'obesità. Dopo un primo infarto del miocardio, questi fattori di rischio continuano sinergicamente a contribuire alla progressione clinica ed alla prognosi della malattia aterosclerotica coronarica.

In pazienti con cardiopatia coronarica stabile, l'attività fisica regolare, oltre a migliorare la capa- 
cità lavorativa e la qualità di vita, riduce il rischio di nuovi eventi coronarici. Il meccanismo cardioprotettivo è multifattoriale. Gli effetti benefici dell'attività fisica regolare sui fattori di rischio coronarico, sulla disfunzione endoteliale, sull' infiammazione, sulla tendenza alla trombosi, sul tono autonomico e sull' ischemia miocardica possono ben contribuire a spiegare la riduzione del rischio.

In considerazione dei benefici clinici e dei ben documentati meccanismi cardioprotettivi, l'attività fisica regolare dovrebbe essere considerata un efficace strumento terapeutico per i pazienti con cardiopatia coronarica.

\section{References}

1. World Health Organization. The World Health Report 2003. www.who.org

2. Pinsky JL, Jette AM, Branch LG, Kannel WB, Feinleib M. The Framingham Disability Study: relationship of various coronary heart disease manifestations to disability in older persons living in the community. Am J Public Health 1990; 80: 363-1367.

3. Ades PA. Cardiac rehabilitation and secondary prevention of coronary heart disease. $N$ Engl J Med 2001; 345: 892-902.

4. Yusuf S, Kawken S, Ounpuu S, et al. Effect of potentially modifiable risk factors associated with myocardial infarction in 52 countries (the INTERHEART study): casecontrol study. Lancet 2004; 364: 937-952.

5. Altieri A, Tavani A, Gallus S, La Vecchia C. Occupational and leisure time physical activity and the risk of nonfatal acute myocardial infarction in Italy. Ann Epidemiol 2004; 14: 461-466.

6. Leon AS, Francklin BA, Costa F, et al. Cardiac rehabilitation and secondary prevention of coronary artery disease. Circulation 2005; 111: 369-376.

7. World Health Organization. Atlas of Heart Disease and Stroke Epidemic. 2004. www.who.org.

8. De Backer G, Ambrosioni E, Borch-Johnsen K, et al. European Guidelines on cardiovascular disease prevention in clinical practice. Europ J of Cardiovasc Prev and Rehab 2003; 10 (suppl 1): S1-S78.

9. Antman EM, Anbe DT, Armstrong PW, et al. ACC/AHA Guidelines for the Management of Patients With ST-Elevation Myocardial Infarction. Circulation 2004 110: e82 - e292.

10. Wenger NK, Froehlicher ES, Smith LK, et al. Cardiac rehabilitation: clinical pratice guidelines. Rockville, Md: Agency for Health Care Policy and Rsearch and the National Heart, Lung, and Blood Institute, 995. (AHCPR publication No. 96-0672).

11. Ades PA, Maloney A, Savage P, Carhart RL Jr. Determinants of physical functioning in coronary patients: response to cardiac rehabilitation. Arch Intern Med 1999; 59: 2357-60.

12. Piña HL, Apstein CS, Balady GJ, et al. Exercise and heart failure. A statement from the American Heart Association Committee on exercise, rehabilitation, and prevention: Circulation 2003; 107: 1210-1225.

13. Myers J, Prakash M, Froelicher V, et al. Exercise capacity and mortality among men referred for exercise testing. N Engl J Med 2002; 346: 793-801.

14. Kavanagh T, Mertens DJ, Hamm LF, et al. Prediction of long-term prognosis in 12169 men referred for cardiac rehabilitation. Circulation 2002; 106: 666-671.

15. Tanasescu M, Leitzmann MF, Rimm EB, Willett WC, Stampfer MJ, Hu FB. Exercise type and intensity in relation to coronary heart disease in men. JAMA 2002; 288: 1994-2000.
16. Manson JE, Greenland P, LaCroix AZ, et al. Walking compared with vigorous exercise for the prevention of cardiovascular events in women. $N$ Engl J Med 2002; 347: 716-725.

17. Lee I-M, Sesso HD, Oguma Y, Paffenbarger RS. Relative intensity of physical activity and risk of coronary heart disease. Circulation 2003; 107: 1110-1116.

18. Kujala UM, Kaprio J, Sarna S, et al. Relationship of leisure-time physical activity and mortality. JAMA 1998; 279: 440-444.

19. Wannamethee SG, Shaper AG, Walker M. Changes in physical acrivity, mortality and incidence of coronary heart disease in older men. Lancet 1998; 351: 1603-1608.

20. Hakim AA, Curb JB, Petrovitch H, et al. Effects of walking on coronary artery disease in elderly men: the Honolulu Heart Program. Circulation 1999; 100: 9-13.

21. Taylor RS, Brown A, Ebrahim S, et al. Exercise-based rehabilitation for patients with coronary heart disease: systematic review and meta-analysis of randomized controlled trials. Am J Med 2004; 116: 682-692.

22. Witt BJ, Jacobsen SJ, Weston SA, et al. Cardiac rehabilitation after myocardial infarction in the community. $J$ Am Coll Cardiol 2004; 44: 988-996.

23. Wannamethee SG, Shaper G, Walker M. Physical activity and mortality in older men with diagnosed coronary heart disease. Circulation 2000; 102: 1358-1363.

24. Lewis EF, Moye LA, Rouleau JL, et al. Predictors of late development of heart failure in stable survivors of myocardial infarction. The CARE study. J Am Coll Cardiol 2003; 42: 1446-1453

25. Belardinelli R, Paolini I, Cianci G, et al. Exercise training intervention after coronary angioplasty: the ETICA trial. J Am Coll Cardiol. 2001; 37: 1891-1900.

26. Hambrecht R, Walther C, Möbius-Winkler S, et al. Percutaneous coronary angioplasty compared with exercise training in patients with stable coronary artery disease. A randomized trial. Circulation 2004; 109: 1371-1378.

27. Giannuzzi P, Temporelli PL, Corrà U, Gattone M, Giordano A, Tavazzi L. Attenuation of unfavorable remodeling by exercise training in postinfarction patients with left ventricular dysfunction. C 1997; 96: 1790-1797.

28. Eckel RH, Grundy SM and Zimmet PZ. The metabolic syndrome. Lancet 2005; 365: 1415-28.

29. Lakk TA, Laaksonen DE, Lakka HM, et al. Sedentary lifestyle. Poor cardio respiratory fitness, and the metabolic syndrome. Med Sci Sports Exerc 2003; 35: 1279-86.

30. Miranda PJ, DeFronzo RA, Califf RM, et al. Metabolic syndrome: Evaluation of pathological and therapeutic outcomes. Am Heart J 2005; 149: 20-32.

31. Bestermann W, Houston MC, Basile J, et al. Addressing the global cardiovascular risk of hypertension, dyslipidemia, diabetes mellitus, and the metabolic syndrome in the southeastern United States, part II: treatment recommendations for management of the global cardiovascular risk of hypertension, dyslipidemia, diabetes mellitus, and the metabolic syndrome. Am J Med Sci 2005; 329 (6): 292-305.

32. Robinson LE, Graham TE. Metabolic syndrome, a cardiovascular disease risk factor: role of adipocytokines and impact of diet and physical activity. Can J Appl Physiol 2004; 29 (6): 808-29.

33. Sundquist K, Qvist J, Johansson SE, Sunquist J. The logterm effect of physical activity on incidence of coronary heart disease: a 12-year follow-up study. Preventive Medicine 2005; 41: 219-25.

34. Hu FB, Stampfer MJ, Solomon C, et al. Physical activity and risk for cardiovascular events in diabetic women. Ann Intern Med 2001; 134: 96-105.

35. Hsia J, Wu LL, Allen C, et al. Physical activity and diabetes risk in postmenopausal women. Am J Prev Med 2005; 28 (1): 19-25.

36. Helmrich SP, Ragland DR, Leung RW, et al. Physical activity and reduced occurrence of non-insulin-dependent diabetes mellitus. N Engl J Med 1991; 325: 147-52. 
37. Lynch J, Helmrich SP, Lakk TA, et al. Moderately intense physical activities and high levels of cardiorespiratory fitness reduce the risk of non-insulin-dependent diabetes mellitus in middle-aged men. Arch Intern Med 1996: 146: 1307-14.

38. Wei M, Gibbons LW, Mitchell TL, et al. The association between cardiorespiratory fitness and impaired fasting glucose and type 2 diabetes mellitus in men. Ann Intern Med 1999; 130: 89-96.

39. Hu G, Qiao Q, Silventoinen K, et al. Occupational, commuting, and leisure-time physical activity in relation to risk for type 2 diabetes in middle-aged Finnish men and women. Diabetologia 2003; 322-29.

40. Tuomilehto J, Lindstrom J, Eriksson JG, et al. Prevention of type 2 diabetes mellitus by changes in lifestyle among subjects with impaired glucose tolerance. $N$ Engl J Med 2001; 344: 1343 - 50.

41. Knowler WC, Barrett-Connor E, Fowler SE, et al. Reduction in the incidence of type 2 diabetes with lifestyle intervention or metformin. N Engl J Med 2002; 346: 393403.

42. Laaksonen DE, Lindstroom J, Lakka TA, et al. Physical activity in the prevention of type 2 diabetes. The Finnish diabetes prevention study. Diabetes 2005; 54: 158-65.

43. Hu G, Jousilahti P, Barengo NC, et al. Physical activity, cardiovascular risk factors, and mortality among Finnish adults with diabetes. Diabetes Care 2005; 28: 799-805.

44. Di Loreto C, Fanelli C, Lucidi P, et al. Make your diabetic patients walk. Long-term impact of different amounts of physical activity on type 2 diabetes. Diabetes Care 2005; 28: 1295-1302.

45. Pescatello LS, Franklin BA, Fabard R, et al. American College of Sports Medicine position stand. Exercise and hypertension. Med Sci Sports Exerc. 2004; 36 (3): 533-53.

46. Hagberg J, Blair S, Ehsani A, et al. Position stand: physical activity, physical fitness, and hypertension. Med Sci Sports Exerc 1993; 25: i-x.

47. Wood P, Stefanich M. Exercise, fitness, and atherosclerosis. In: Bouchard C, ed. Exercise, Fitness, and Health. Champaign, Ill: Human Kinetics; 1988: 409-423.

48. Dengel DR, Galecki AT, Hagberg JM, Pratley RE. Independent and combined effects of weight loss and aerobic exercise on blood pressure and oral glucose tolerance in older men. Am J Hypertension 1998; 11: 1405-12.

49. Hamberg JM, f RE, Dengel DR, Wilund KR. Exercise training-induced blood pressure and plasma lipid improvements in hypertensives may be genotype dependent. Hypertension 1999; 34: 18-23.

50. Hu G, Tuomilehto J, Silventoinen K, et al. Joint effects of physical activity, body mass index, waist circumference and waist-to-hip ratio with the risk of cardiovascular disease among middle-aged Finnish men and women. European Heart Journal 2004; 25: 2212-19.

51. Christou DD, Gentile CL, Desouza A, et al. Fatness is a better predictor of cardiovascular disease risk factor profile than aerobic fitness in healty men. Circulation 2005; 111: 1904-14.

52. Garrow JS, Summerbell CD. Meta-analysis: effect of exercise, with or without dieting, on the body composition of overweight subjects. Eur J Clin Nutr 1995; 49: 1.

53. King AC, Tribble DL. The role of exercise in weight regulation in non-athletes. Sports Med 1991; 11: 331.34.

54. Manson JE, Willet WC, Stampfer MJ, et al. Body weight and mortality among women. $N$ Engl J Med 1995; 333: 677-85.

55. Hu FB, Willett WC, Li T, et al. Adiposity as compared with physical activity in predicting mortality among women. N Engl J Med 2004; 351: 2694-703.

56. Wilmore JH. Weight gain, weight loss, and weight control: What is the role of physical activity? Nutrition 1997; 13 (9): 820-22.

57. Grundy SM, Cleeman JI, Merz CNB et al.Implications of Recent Clinical Trials for the National Cholesterol Edu- cation Program Adult Treatment Panel III Guidelines. $J$ Am Coll Cardiol 2004; 44: 720-32.

58. Kelley GA, Kelly KS, and Tran ZV. Walking, lipids, and lipoproteins: a meta-analysis of randomized controlled trials. Preventive Medicine 2004; 38: 651-61.

59. Kraus WE, Houmard JA, Dusha BD, et al. Effects of the amount and intensity of exercise on plasma lipoproteins. N Engl J Med 2002; 347, 19: 1483.92.

60. Levine GN, Keaney JF Jr, Vita JA. Cholesterol reduction in cardiovascular disease: clinical benefits and possible mechanisms. N Engl J Med 1995; 332: 512-521.

61. Widlansky ME, Gokce N, Keaney JF, Vita JA. The clinical implications of endothelial dysfunction. $J$ Am Coll Cardiol 2003; 42: 1149-1160.

62. Libby P. Current concepts of the pathogenesis of the acute coronary syndromes. Circulation 2001; 104: 365-372.

63. Cooke JP, Losordo DW. Nitric oxide and angiogenesis. Circulation 2002; 105: 2133-2135.

64. Dimmeler S, Zeiher AM. Endothelial cell apoptosis in angiogenesis and vessel regression. Circ Res 2000; 87: 434439.

65. Vita JA, Keaney JF. Endhotelial function. A barometer for cardiovascular risk? Circulation 2002; 106: 640-642.

66. Lerman A, Zeiher AM. Endothelial function. Cardiac events. Circulation 2005; 111: 363-368.

67. Heitzer T, Schlinzig T, Khron K, Meinertz T, Munzel T. Endothelial dysfunction, oxidative stress, and risk of cardiovascular events in patients with coronary artery disease. Circulation 2001; 104: 2673-2678.

68. Fichtlscherer S, Breuer S, Zeilher AM. Pognostic value of systemic endothelial dysfunction in patients with acute coronary syndromes. Further evidence for the existence of the "vulnerable" patients. Circulation 2004; 110 1926-1932.

69. Targonski PV, Bonetti PO, Pumper GM, Higano ST, Holmes DR, Lerman A. Coronary endothelial dysfunction is associated with an increased risk of cerebrovascular events. Circulation 2003; 107: 2805-2809.

70. Kitta Y, Nakamura T, Kodama Y, et al. Endothelial vasomotor dysfunction in the brachial artery is associated with late in-stent coronary restenosis. J Am Coll Cardiol 2005 ; in press.

71. Hambrecht R, Wolf A, Gielen S, et al. Effect of exercise on coronary endothelial function in patients with coronary artery disease. $N$ Engl J Med 2000; 342: 454-460.

72. Vona M, Rossi A, Capodaglio P, et al. Impact of physical training and detraining on endothelium-dependent vasodilation in patients with recent acute myocardial infarction. Am Heart J 2004; 147: 1039-1046.

73. Dimmeler S, Hermann C, Galle J, et al. Upregulation of superoxide dismutase and nitric oxide synthase mediates the apoptosis-suppressive effects of shear stress on endothelial cells. Arterioscler Thromb Vasc Biol 1999; 19: 656-664.

74. Sessa WC, Prichard K, Seyedi N, et al. Chronic exercise in dogs increases coronary vascular nitric oxide production and endothelial cell nitric oxide synthase gene expression. Circ Res 1994; 74: 349-353.

75. Hambrecht R, Adams V, Erbs S, et al. Regular physical activity improves endothelial function in patients with coronary artery disease by increasing phosphorylation of endothelial nitric oxide synthase. Circulation 2003; 103: 3152-3158.

76. Grieldling KK, Sorescu D, Ushio-Fukai M. NAD(P)H oxidase: role in cardiovascular biology and disease. Circ Res 2000; 86: 494-501.

77. Gölach A, Brandles RP, Nguyen K, Amidi M, Dehghani F, Busse R. A gp91phox containing NADPH oxydase selectively expressed in endothelial cells is a major source of oxygen radical generation in the arterial wall. Circ Res 2000; 87: 26-32.

78. Adams V, Linke A, Kränkel N, et al. Impact of regular physical activity on the $\mathrm{NAD}(\mathrm{P}) \mathrm{H}$ oxydase and angiotensin 
receptor system in patients with coronary artery disease. Circulation 2005; 111: 555-562.

79. Szmitko PE, Fedak PWM, Weisel RD, Stewart DJ, Kutryk MJB, Verma S. Endothelial progenitor cells. New hope for a broken heart. Circulation 2003; 107: 30933100 .

80. Crosby JR, Kaminski WE, Schatteman G, et al. Endothelial cells of hematopoietic origin make a significant contribution to adult blood vessel formation. Circ Res 2000; 87: 728-730

81. Schmidt-Lucke C, Rössig, Fichtlscheree S, et al. Reduced number of circulating endothelial progenitor cells predicts future cardiovascular events. Proof of concept for the clinical importance of endogenous vascular repair. Circulation 2005; 111: 2981-2987.

82. Laufs U, Werner N, Link A, et al. Physical training increases endothelial progenitor cells, inhibits neointima formation, and enhances angiogenesis. Circulation 2004; 109: 220-226.

83. Sandri M, Adams V, Gielen S, et al. Effects of exercise and ischemia on mobilization and functional activation of blood-derived progenitor cells in patients with ischemic syndromes. Results of 3 randomized studies. Circulation 2005; 111: 3391-3399.

84. Libby P, Ridker PM, Maseri A. Inflammation and atherosclerosis. Circulation 2002; 105: 1135-1143.

85. Verma S, Buchanan MR, Anderson TJ. Endothelial function testing as a biomarker of vascular disease. Circulation 2003; 108: 2054-2059.

86. Biasucci LM. Clinical use of inflammatory markers in patients with cardiovascular diseases. Circulation 2004; 110: e560-e567.

87. Milazzo D, Biasucci LM, Luciani N, et al. Elevated levels of C-reactive protein before coronary artery bypass grafting predict recurrence of ischemic events. Am J Cardiol 1999; 84: 459-461.

88. Zouridakis E, Avanzas P, Arroyo-Espliguero R, Fredericks S, Kaski JC. Markers of inflammation and rapid coronary artery disease progression in patients with stable angina pectoris. Circulation 2004; 110: 1747-1753.

89. Kasapis C, Thompson PD. The effects of physical activity on serum C-reactive protein and inflammatory markers. A systematic review. J Am Coll Cardiol 2005; 45: 1563-1569.

90. Milani RV, Lavie CJ, Mehra MR. Reduction in C-reactive protein through cardiac rehabilitation and exercise training. J Am Coll Cardiol 2004; 43: 1056-1061.
91. Tisi PV, Hulse M, Chulakadabba A, Gosling P, Shearman $\mathrm{CP}$. Exercise training for intermittent claudication: does it adversely affect biochemical markers of the exercise-induced inflammatory response? Eur J Vasc Endovasc Surg 1997; 14: 344-350.

92. Berg AH, Scherer PE. Adipose tissue, inflammation, and cardiovascular disease. Circ Res 2005; 96: 939-949.

93. Lee KW, Lip GYH. Effects of lifestyle on hemostasis, fibrinolysis, and platelet reactivity. A systematic review. Arch Intern Med 2003; 163: 2368-2392.

94. Imhof A, Koenig W. Exercise and thrombosis. Cardiol Clin 2001; 19: 389-400.

95. Smith DT, Hoetzer GL, Greiner JJ, Stauffer BL, DeSouza CA. Effects of ageing and regular aerobic exercise on endothelial fibrinolytic capacity in humans. J Physiol 2003; 546: 289-298.

96. Bigger JT Jr, Fleiss JL, Steinman RC, Rolnitzky LM, Kleiger RE, Rottman JN. Frequency domain measures of heart period variability and mortality after myocardial infarction. Circulation 1992; 85: 164-171.

97. LaRovere MT, Bigger JT Jr, Marcus FI, Mortara A, Schwartz PJ. Baroreflex sensitivity and heart rate variability in prediction of total cardiac mortality after myocardial infarction. Lancet 1998; 351: 478-484.

98. Billman GE. Aerobic exercise conditioning: a nonpharmacologic antiarrhythmic intervention. $J$ Appl Physiol 2002; 92: 446-454.

99. Iellamo F, Legramante JM, Massaro M, Raimondi G, Galante A, Effects of a residential exercise training on baroreflex sensitivity and heart rate variability in patients with coronary artery disease. A randomized, controlled study. Circulation 2000; 102: 2588-2592.

100. Malfatto G, Facchini M, Sala L, Branzi G, Bragato R, Leonetti G. Effects of cardiac rehabilitation and betablocker therapy on heart rate variability after first acute myocardial infarction. Am J Cardiol 1998; 81: 834-840.

101. Leitch JW, Newling RP, Basta M, et al. Randomized trial of a hospital-based exercise training program after acute myocardial infarction: cardiac autonomic effects. $J$ Am Coll Cardiol 1997; 29: 1263-1268.

102. Shepard RJ, Balady GJ. Exercise as cardiovascular therapy. Circulation 1999; 99: 963-972.

103. Belardinelli R, Georgiou D, Ginzton L, Cianci G, Purcaro A. Effects of moderate exercise training on thallium uptake and contractile response to low-dose dobutamine of dysfunctional myocardium in patients with ischemic cardiomyopathy. Circulation 1998; 97: 553-561.

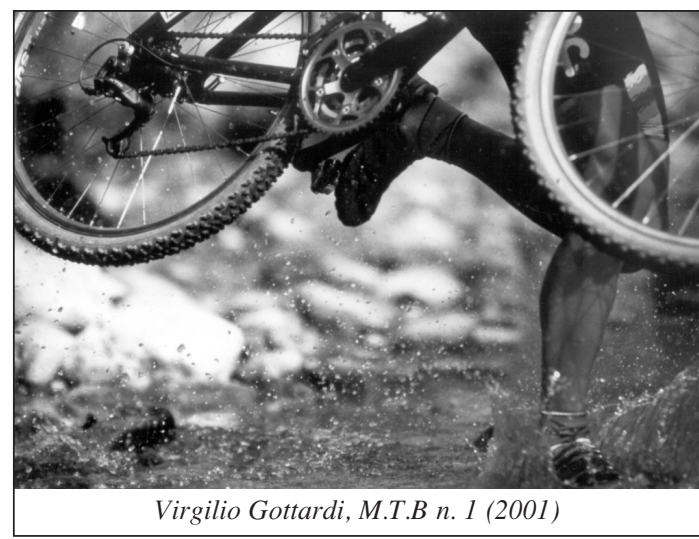

